\title{
Composição e diversidade de anfíbios anuros em dois ambientes de Mata Atlântica no Parque Estadual Carlos Botelho, São Paulo, sudeste do Brasil
}

\author{
Renato Augusto de Moraes ${ }^{1,}$, Ricardo J. Sawaya ${ }^{2}$ \& Walter Barrella ${ }^{3}$
}

Biota Neotropica v7(n2) - http://www.biotaneotropica.org.br/v7n2/pt/abstract?article+bn00307022007

\author{
Recebido em 09/11/06 \\ Versão reformulada recebida em 15/01/07 \\ Publicado em 31/03/07
}

\author{
${ }^{1}$ Departamento de Ecologia, Instituto de Biociências, Universidade de São Paulo - USP, \\ Rua do Matão, Travessa 14, n. 321, CEP 05508-900, São Paulo, SP, Brasil \\ ${ }^{2}$ Laboratório de Herpetologia, Instituto Butantan, Av. Doutor Vital Brazil, 1500, \\ CEP 05503-900, São Paulo, SP, Brasil, e-mail: sawaya@butantan.gov.br \\ ${ }^{3}$ Departamento de Ciências do Ambiente, Pontifícia Universidade Católica de São Paulo - PUC-SP, \\ Praça José Ermírio de Moraes, 290, CEP 18030-230, \\ Sorocaba, SP, Brasil, e-mail: barrella@ sorocaba.pucsp.br \\ Autor para correspondência: Renato Augusto de Moraes, e-mail: ramoraes.reptilia@gmail.com
}

\begin{abstract}
Moraes, R.A. de, Sawaya, R.J. \& Barrela, W. Composition and diversity of A nuran Amphibians in two Atlantic Forest environments in Southeastern Brazil, Parque Estadual Carlos Botelho, São Paulo, Brazil. Biota Neotrop. May/Aug 2007 vol. 7, no. 2 http://www.biotaneotropica.org.br/v7n2/pt/abstract?article+bn0030 7022007. ISSN 1676-0603.

The Atlantic Forest is characterized by its high species richness and endemism, and is one of the 25 hotspots of biodiversity around the world. We present information on composition and diversity of anuran amphibians in two environments with different degrees of disturbance in Parque Estadual Carlos Botelho, São Paulo state, an area of Atlantic Rain Forest in southeastern Brazil. We sampled an artificial pond in a disturbed area and a pond in a preserved area by audio sampling and incidental encounters, in 36 nights in each habitat. We compared the species composition, richness, dominance, and relative abundance between the two habitats. Despite distant only ca. $400 \mathrm{~m}$, we recorded only two species in common between the two habitats. We recorded in the disturbed area 10 species $(\mathrm{N}=518)$ with $46.7 \%$ of dominance of Hypsiboas albopunctatus; and in the preserved habitat we recorded 11 species $(\mathrm{N}=656)$ with $36.0 \%$ of dominance of Dendropsophus giesleri. Richness was not significantly different between the habitats. The estimated dominance by rarefaction in the preserved habitat was significantly lower than that in the disturbed habitat. The capability of some species populations in colonizing the disturbed habitat, the distinct physiological tolerance among species, and the dependence on specific microhabitats for reproduction are possible factors related to the observed patterns. We suggest that additional alterations of the Atlantic Forest cover inside the reserve might result in a decrease of alpha-diversity by eliminating forest dependent species, as well as a consequently increase in beta-diversity by the colonization of open area species that are ecologically more generalist. The slightly higher richness and mainly lower dominance observed in the preserved area indicate it is the habitat with higher species diversity. Thus, the presence of old-growth and/or conserved forests in the reserve is essential to maintain the local anuran diversity.

Keywords: Amphibia, biodiversity, colonizing species, conservation, dominance, relative abundance, species richness, tropical forest.
\end{abstract}

\section{Resumo}

Moraes, R.A. de, Sawaya, R.J. \& Barrela, W. Composição e diversidade de anfíbios anuros em dois ambientes de Mata Atlântica no Parque Estadual Carlos Botelho, São Paulo, sudeste do Brasil. Biota Neotrop. May/Aug 2007 vol. 7, no. 2 http://www.biotaneotropica.org.br/v7n2/pt/abstract?article+bn00307022007. ISSN 1676-0603.

A Mata Atlântica é caracterizada pela elevada riqueza e endemismo de espécies, e é um dos 25 hotspots de biodiversidade mundiais. Apresentamos informações sobre a composição e diversidade de espécies de anfíbios anuros em dois ambientes com diferentes graus de perturbação no Parque Estadual Carlos Botelho, SP, uma área de Mata Atlântica no sudeste do Brasil. Amostramos um açude em área alterada e uma lagoa em área preservada, através de procura auditiva e encontros ocasionais em 36 noites de observação em cada ambiente. As duas áreas foram comparadas em relação à composição, riqueza, dominância e abundância relativa de espécies. Apesar de próximos, cerca de $400 \mathrm{~m}$, apenas duas espécies foram comuns aos dois ambientes. Na área alterada registramos 10 espécies $(\mathrm{N}=518)$ e dominância de 46,7\% de Hypsiboas albopunctatus, e na área preservada 11 espécies ( $N=656$ ) e dominância de $36,0 \%$ de Dendropsophus giesleri. A riqueza não foi significativamente diferente nos dois ambientes. A dominância estimada por rarefação na área preservada foi significativamente menor em 
relação à observada na área alterada. A capacidade das populações de algumas espécies em colonizar a área alterada, tolerâncias fisiológicas distintas entre as espécies e a dependência de microambientes específicos para a reprodução são possíveis fatores responsáveis pelos padrões observados. Sugerimos que alterações adicionais da cobertura original de Mata Atlântica na reserva poderão resultar em um decréscimo na diversidade alfa, devido a eliminação de espécies ecologicamente dependentes de ambientes florestais, e um conseqüente aumento na diversidade beta, resultante da colonização de espécies de área alterada ecologicamente mais generalistas. A riqueza ligeiramente maior e principalmente a menor dominância observada na área preservada indicam ser esta uma área que apresenta maior diversidade de espécies. Assim, a presença de florestas pouco perturbadas e/ou conservadas na reserva é fundamental para a manutenção da diversidade da anurofauna local.

Palavras-chave: abundância relativa, Amphibia, biodiversidade, conservação, dominância, espécies colonizadoras, floresta tropical, riqueza de espécies.

\section{Introdução}

A região Neotropical possui a maior riqueza em espécies de anfíbios anuros do mundo (Duellman 1988, 1999). Isto é particularmente verdadeiro para o Estado de São Paulo, sudeste do Brasil, que abriga cerca de 35\% dos táxons descritos para todo o país (Haddad 1998). Entre as formações vegetais existentes no sudeste brasileiro, certamente a Mata Atlântica é um dos ecossistemas que mais contribuem para este quadro, pois além de proporcionar inúmeros microambientes que favorecem a evolução de modos reprodutivos especializados, apresenta grande número de espécies endêmicas, o que deve estar relacionado à topografia acidentada de seu terreno (Haddad 1998, Haddad \& Prado 2005).

As florestas tropicais brasileiras têm sido continuamente destruídas e fragmentadas nas últimas décadas (Gascon et al. 2000, Olmos \& Galetti 2004), mas ainda são escassos os estudos que abordam aspectos comparativos da composição e diversidade de anuros entre áreas próximas em diferentes estados de conservação nestas localidades (e.g., Lieberman 1986, Heyer et al. 1990, Heinen 1992, Tocher 1998, Bernarde et al. 1999, Neckel-Oliveira et al. 2000). Parece haver um consenso de que áreas florestais secundárias dentro de uma mesma região possuem menor diversidade de espécies em relação a áreas florestais pouco perturbadas, além de ambas diferirem de um modo geral na composição e nos padrões de dominância e abundância relativa de espécies da anurofauna (Lieberman 1986, Heinen 1992, Tocher 1998, Machado et al. 1999, Neckel-Oliveira et al. 2000).

O tipo de cobertura vegetal e/ou o período desde a perturbação do ambiente são alguns dos fatores apontados como possíveis responsáveis por estas diferenças na diversidade e composição de espécies entre estas áreas (Inger \& Colwell 1977, Heinen 1992, Tocher 1998). O estudo destas relações é importante, porque fornece subsídios para a conservação das comunidades naturais e auxilia na compreensão dos processos envolvidos na restauração da fauna após a perturbação de um ambiente (e.g., Heinen 1992, Pearman 1997).

Durante as últimas décadas é crescente o número de relatos sobre declínios e extinções populacionais de anfíbios em muitas regiões do mundo (e.g., Pechmann \& Wilbur 1994, Blaustein \& Wake 1995, Houlahan et al. 2000, Collins \& Storfer 2003), inclusive em áreas de Mata Atlântica (Heyer et al. 1988, Weygoldt 1989, Guix et al. 1998, Pombal-Jr \& Haddad 1999, Eterovick et al. 2005). No sudeste do Brasil, o desmatamento de áreas naturais parece ser uma das principais causas destes declínios e, certamente, algumas espécies de anuros foram extintas antes que um especialista pudesse ter acesso a alguns exemplares (Haddad 1998, Pombal-Jr \& Gordo 2004). Estes fatores, aliados ao relativo desconhecimento sobre ecologia, história natural, diversidade e status de conservação da fauna de anuros na região Neotropical (Caldwell 1996, Azevedo-Ramos \& Galatti 2002, Pombal-Jr \& Gordo 2004, Zaher et al. 2005) demandam a urgência de estudos desta natureza na região.
A Mata Atlântica é considerada um dos 25 hotspots mundiais de biodiversidade, sendo reconhecida por sua excepcional riqueza e endemismo de espécies (Myers et al. 2000, Silva \& Casteleti 2005). Atualmente, com apenas cerca de $8 \%$ de sua área original (Morellato $\&$ Haddad 2000), esse bioma consiste em sua maior parte de pequenos fragmentos florestais isolados e a intensa intervenção antrópica continua a colocar em risco a sua biodiversidade (Olmos \& Galetti 2004, Hirota 2005). A perda da biodiversidade causa alterações nos serviços e produtos providos pelos ecossistemas o que afeta a vida e a subsistência da população humana, além de contribuir para a ruptura e esgotamento de processos evolutivos (Ehrlich \& Wilson 1991, Myers \& Knoll 2001).

Com o objetivo de contribuir para o conhecimento sobre a biodiversidade e conservação dos remanescentes florestais de Mata Atlântica do Estado de São Paulo, este estudo caracteriza a composição e diversidade (riqueza, dominância e abundância relativa de espécies) de espécies de anfíbios anuros em dois ambientes com diferentes graus de perturbação no Parque Estadual Carlos Botelho, Estado de São Paulo.

\section{Material e Métodos}

\section{1. Área de estudo}

A amostragem foi realizada em dois ambientes a cerca de $800 \mathrm{~m}$ de altitude no Parque Estadual Carlos Botelho (PECB; $24^{\circ} 01^{\prime}$ a 24 $13^{\prime}$ S e $47^{\circ} 47^{\prime}$ a $48^{\circ} 06^{\prime}$ O; (Figura 1), no município de São Miguel Arcanjo, Estado de São Paulo, sudeste do Brasil. O PECB está localizado na região sul do Estado de São Paulo, ao longo da Escarpa Atlântica, na Serra de Paranapiacaba, e apresenta área total de 37.644 ha (Ferraz \& Varjabedian 1999). A formação vegetal predominante é de Floresta Atlântica Submontana (Oliveira-Filho \& Fontes 2000), genericamente conhecida como Mata Atlântica. Segundo a classificação de Köppen (1948), a região do PECB situada nas partes mais elevadas da serra apresenta o clima do tipo $\mathrm{Cfb}$, ou úmido sem estiagem. A pluviosidade atinge de 1700 a $2000 \mathrm{~mm}$ anuais e a média da temperatura anual oscila entre 17 e $18{ }^{\circ} \mathrm{C}$ (Camargo et al. 1972).

Foi amostrado um açude com cerca de $20.000 \mathrm{~m}^{2} \mathrm{em}$ área de mata secundária (área alterada), que foi construído após o desmatamento de uma área natural de Mata Atlântica, a cerca de 25 anos, com a finalidade de captar água para a sede do Parque. Também foi amostrada uma poça permanente com aproximadamente $500 \mathrm{~m}^{2}$ de área em ambiente pouco perturbado de Mata Atlântica (área preservada). A vegetação marginal do açude é constituída por plantas arbustivas, herbáceas pioneira e uma plantação de Araucaria angustifolia (Figura 2a), enquanto a vegetação no entorno da poça permanente é constituída 


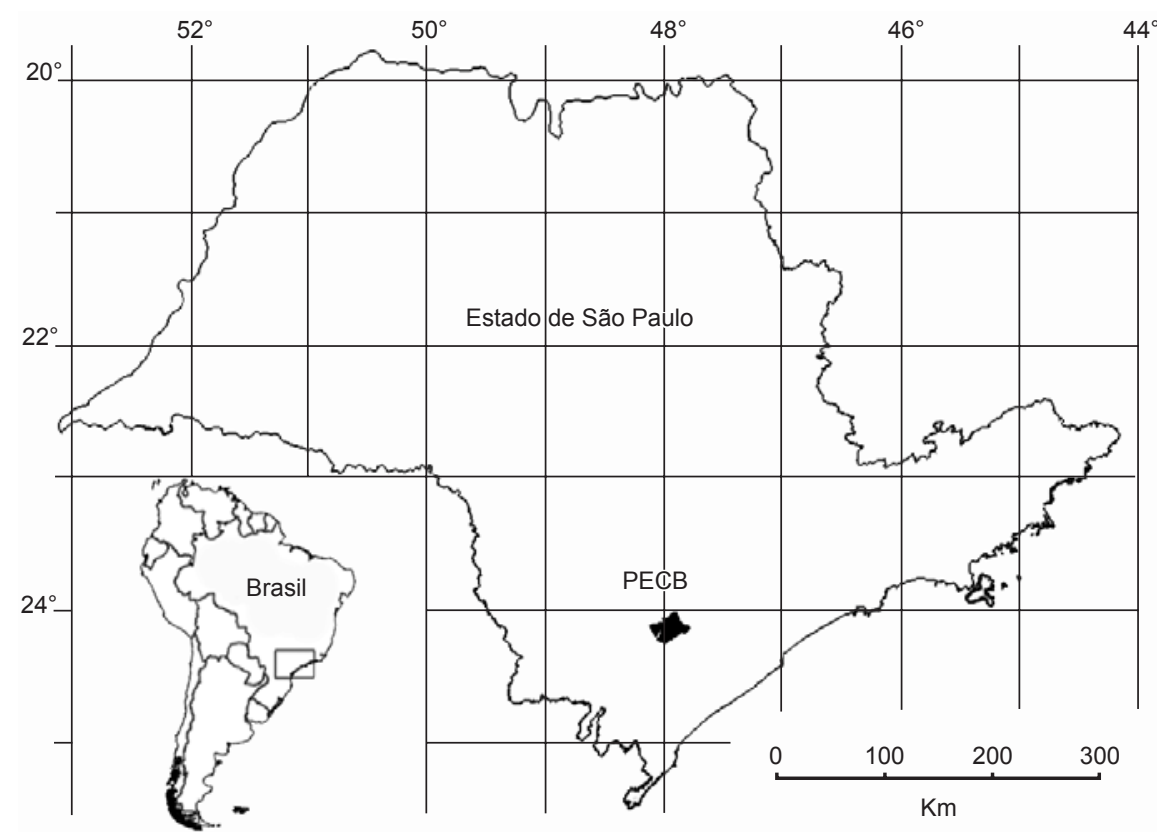

Figura 1. Localização da área do Parque Estadual Carlos Botelho (PECB) no Estado de São Paulo, sudeste do Brasil.

Figure 1. Location of Parque Estadual Carlos Botelho (PECB) in São Paulo state, southeastern Brazil.

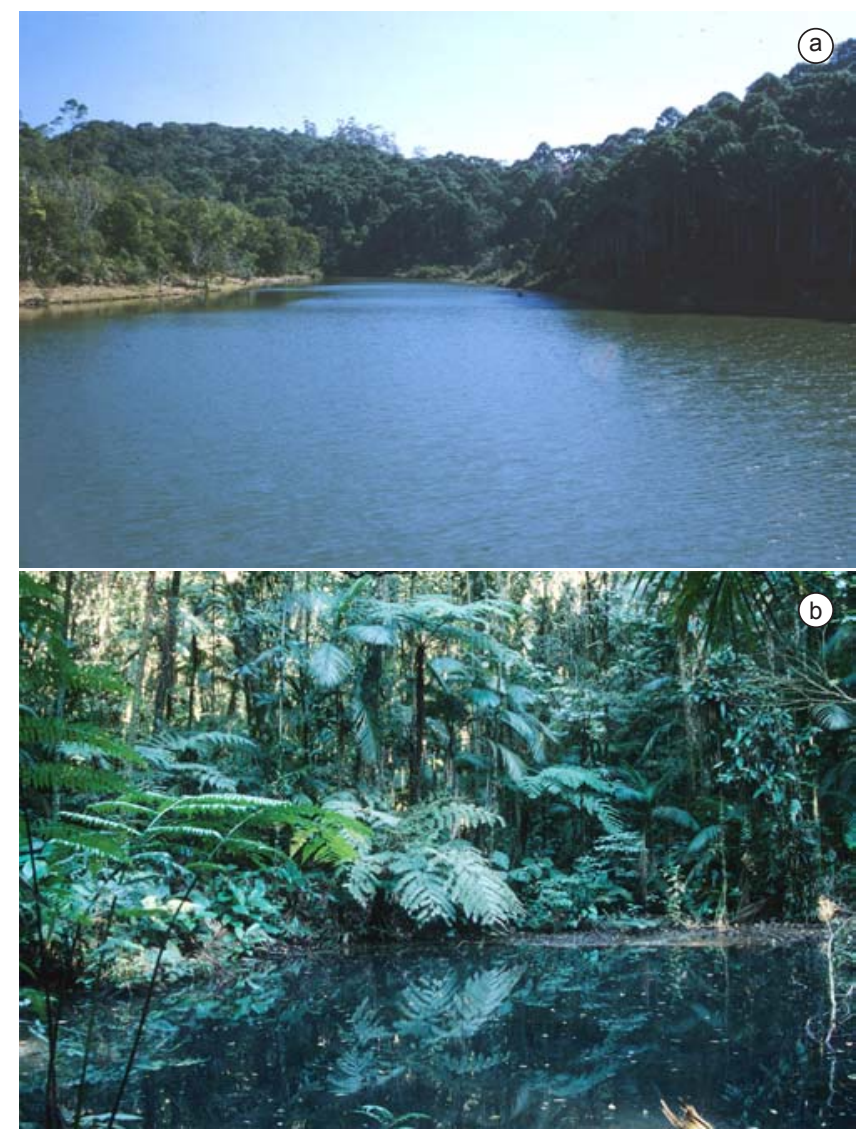

Figura 2. Ambientes amostrados no Parque Estadual Carlos Botelho: a) área alterada, açude em área de mata secundária; b) área preservada, poça permanente em mata pouco perturbada.

Figure 2. Habitats sampled in Parque Estadual Carlos Botelho (PECB): a) disturbed habitat, artificial pond in secondary forest; b) preserved habitat, permanent pond in old-growth forest. por plantas arbóreas e arbustivas, ricas em epífitas (Figura 2b). A distância entre os dois ambientes é de cerca de $400 \mathrm{~m}$.

\section{Coleta de dados}

A coleta regular de dados foi realizada entre agosto de 1999 e julho de 2000, em amostragens quinzenais de uma ou duas noites em cada um dos ambientes, de forma alternada, totalizando 36 noites de observação em cada ambiente. As observações iniciaram-se pouco antes do pôr-do-sol (ca. de 18 horas) até cerca de 01:00 hora.

Os seguintes métodos de amostragem foram utilizados: procura auditiva e encontros ocasionais. A procura auditiva foi realizada de acordo com o método "audio strip transect" (cf. Heyer et al. 1994), mas limitada ao entorno dos corpos d'água. Os encontros ocasionais consistiram na contagem de indivíduos machos que não vocalizavam na área de estudo durante a amostragem, como registro fotográfico e coleta dos espécimes. Fêmeas foram registradas apenas eventualmente durante os encontros ocasionais. A abundância relativa de indivíduos de cada espécie foi estimada principalmente a partir da atividade de vocalização dos machos, registrados no horário de pico de vocalização em cada noite de trabalho. A abundância das espécies para as quais os machos não apresentaram atividade de vocalização foi estimada através de encontros ocasionais.

Dois espécimes-testemunho de cada espécie encontrada foram coletados e transportados em sacos plásticos umedecidos e levados para a sede do Parque, onde foram mortos em álcool 10\%, fixados em formol $10 \%$ e conservados em álcool $70 \%$. Sempre que possível, foram fotografados em seus sítios de vocalização ou de reprodução. Em alguns casos, a ocorrência da espécie também foi registrada por meio da gravação de sua vocalização. Os espécimes foram depositados na Coleção Herpetológica Richard Alphonse Hoge (IBSP), Instituto Butantan, São Paulo, São Paulo, Brasil.

\section{Análise dos dados}

Para analisar a riqueza de espécies de anuros em cada ambiente, foram confeccionadas curvas de rarefação de espécies (senso Gotelli \& Colwell 2001) com o programa EstimateS 7.5.0 (Colwell 2005), 
e 1000 aleatorizações. O programa gera 1000 curvas de acumulação de espécies aleatorizando a ordem das amostras; assim, cada ponto da curva corresponde à média de riqueza acumulada nas 1000 curvas e está associado a um desvio-padrão. Cada noite de observação foi considerada uma amostra, o que resultou em 36 amostras para cada ambiente. Também foram confeccionadas curvas de rarefação de espécies em relação ao número de indivíduos capturados em cada ambiente.

A comparação de taxocenoses com números diferentes de indivíduos capturados pode levar a conclusões errôneas, uma vez que o número de espécies está relacionado ao número de indivíduos capturados (Melo et al. 2003, Begon et al. 2006). Assim, devido ao maior número de indivíduos amostrados na área preservada (656), em relação à área alterada (518), foi utilizado o método da rarefação (Sanders 1968, Krebs 2000) para comparar a riqueza considerando o mesmo número de indivíduos, correspondente ao tamanho da menor amostra, ou 518 indivíduos. A dominância nos dois ambientes, representada pelo índice de Berger-Parker (Magurran 1988), ou simplesmente a porcentagem da espécie mais abundante, também foi comparada entre os dois ambientes através do mesmo método. Para estas análises foi utilizado o programa ECOSIM 7.0 (Gotelli \& Entsminger 2001). O programa permite fixar um número de indivíduos menor do que o observado em uma amostra, e estima a riqueza e a dominância para este número de indivíduos retirados aleatoriamente da amostra. Para as estimativas, foram realizadas 1000 aleatorizações, sendo o resultado uma média das 1000 possibilidades e intervalo de confiança de $95 \%$.

A riqueza de espécies entre os ambientes também foi comparada pelo estimador Jacknife 1 (Burnham \& Overton 1979), calculado no programa EstimateS 7.5.0 (Colwell 2005). Para a escolha do estimador de riqueza, foram realizadas comparações visuais entre curvas de rarefação de seis estimadores: Chao 1 e 2; Jacknife 1 e 2; ACE e ICE. O estimador Jacknife 1 foi escolhido por apresentar o melhor comportamento ao longo da acumulação das amostras nos dois ambientes.

A distribuição de abundância das espécies utiliza toda a informação obtida em uma comunidade e é a descrição matemática mais completa dos dados (Magurran 1988). Assim, além da dominância, comparamos visualmente a abundância relativa de espécies dos dois ambientes através de gráficos de colunas.

\section{Resultados}

Foram registrados nos dois ambientes 1.174 indivíduos de quatro famílias, sete gêneros e 19 espécies (Tabela 1; Figura 3). Apesar de bastante próximos (ca. $400 \mathrm{~m}$ ), os dois ambientes amostrados apresentaram composição de espécies diferente (Tabela 1). Das 11 espécies

Tabela 1. Lista de espécies, número de indivíduos e porcentagem de anfíbios anuros encontrados no ambiente de área alterada (açude, mata secundária) e área preservada (poça permanente, mata pouco perturbada) do Parque Estadual Carlos Botelho, SP, entre agosto de 1999 e julho de 2000.

Table 1. Species list, number of individuals and percentage of amphibian anurans found in the disturbed habitat (artificial pond, secondary forest) and preserved habitat (permanent pond, old-growth forest) of Parque Estadual Carlos Botelho, SP, between August 1999 and July 2000.

\begin{tabular}{|c|c|c|c|c|c|}
\hline \multirow[t]{3}{*}{ Família } & \multirow[t]{3}{*}{ Espécie } & \multicolumn{4}{|c|}{ Ambiente } \\
\hline & & \multicolumn{2}{|c|}{ Área alterada } & \multicolumn{2}{|c|}{ Área preservada } \\
\hline & & $\mathbf{N}$ & $\%$ & $\mathbf{N}$ & $\%$ \\
\hline \multicolumn{6}{|l|}{ BUFONIDAE } \\
\hline & Chaunus ictericus & 61 & 11,8 & - & - \\
\hline & Chaunus ornatus & 30 & 5,8 & - & - \\
\hline \multicolumn{6}{|l|}{ HYLIDAE } \\
\hline & Dendrophryniscus brevipollicatus & - & - & 8 & 1,2 \\
\hline & Aplastodiscus albosignatus & - & - & 79 & 12 \\
\hline & Dendropsophus giesleri & - & - & 236 & 36 \\
\hline & Dendropsophus minutus & 15 & 2,9 & 2 & 0,3 \\
\hline & Hypsiboas albopunctatus & 242 & 46,7 & - & - \\
\hline & Hypsiboas bischoffi & 35 & 6,8 & 68 & 10,4 \\
\hline & Hypsiboas faber & 27 & 5,2 & - & - \\
\hline & Hypsiboas prasinus & 75 & 14,5 & - & - \\
\hline & Scinax cf. ruber & - & - & 18 & 2,7 \\
\hline & Scinax cf. catharinae & - & - & 2 & 0,3 \\
\hline & Scinax fuscomarginatus & 2 & 0,4 & - & - \\
\hline & Scinax hayii & - & - & 32 & 4,9 \\
\hline & Scinax perereca & - & - & 55 & 8,4 \\
\hline \multicolumn{6}{|l|}{ LEIUPERIDAE } \\
\hline & Physalaemus cuvieri & 29 & 5,6 & - & - \\
\hline & Physalaemus olfersii & - & - & 105 & 16 \\
\hline \multicolumn{6}{|c|}{ LEPTODACTYLIDAE } \\
\hline & Leptodactylus cf. ocellatus & 2 & 0,4 & - & - \\
\hline & Proceratophrys boiei & - & - & 51 & 7,8 \\
\hline & & 518 & - & 656 & - \\
\hline
\end{tabular}



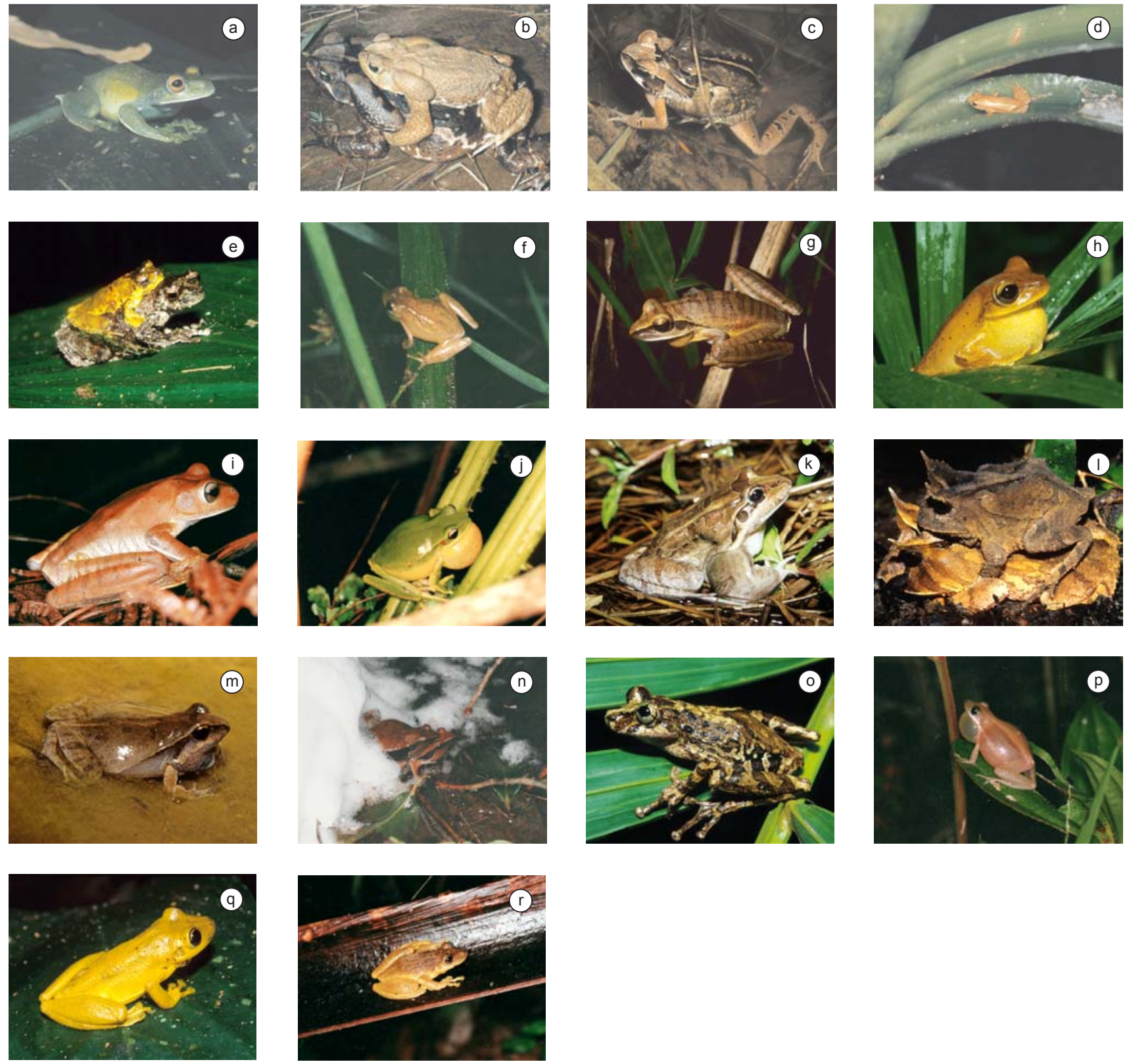

Figura 3. Espécies de anfíbios anuros encontradas no Parque Estadual Carlos Botelho, SP, a cerca de $800 \mathrm{~m}$ de altitude nos dois ambientes estudados, entre agosto de 1999 e julho de 2000. a) Aplastodiscus albosignatus; b) Chaunus ictericus; c) C. ornatus; d) Dendrophryniscus brevipollicatus; e) Dendropsophus giesleri; f) Dendropsophus minutus; g) Hypsiboas albopunctatus; h) H. bischoff; i) H. faber; j) H. prasinus; k) Leptodactylus cf. ocellatus; 1) Proceratophrys boiei; m) Physalaemus cuvieri; n) Physalaemus olfersii; o) Scinax cf. catharinae; p) Scinax fuscomarginatus; q) S. hayii e r) Scinax perereca. Fotos: R. A. Moraes (exceto 3Q: L. M. Castanho).

Figure 3. Amphibian anuran species found in Parque Estadual Carlos Botelho, SP, between August 1999 and July 2000. a) Aplastodiscus albosignatus; b) Chaunus ictericus; c) C. ornatus; d) Dendrophryniscus brevipollicatus; e) Dendropsophus giesleri; f) Dendropsophus minutus; g) Hypsiboas albopunctatus; h) H. bischoff; I) H. faber; j) H. prasinus; k) Leptodactylus cf. ocellatus; 1) Proceratophrys boiei; m) Physalaemus cuvieri; n) Physalaemus olfersii; o) Scinax cf. catharinae; p) Scinax fuscomarginatus; q) S. hayii and r) Scinax perereca. Photos: R. A. Moraes (except 3Q: L. M. Castanho).

registradas na área preservada, nove foram exclusivas deste ambiente. Das 10 espécies registradas na área alterada, oito foram observadas apenas neste ambiente. Somente duas espécies ocorreram nos dois ambientes: Hypsiboas bischoffi e Dendropsophus minutus.

Além da diferença na composição, o padrão de abundância das espécies diferiu nos dois ambientes (Tabelas 1 e 2; Figura 4).

$\mathrm{Na}$ área alterada foram registrados 518 indivíduos pertencentes a quatro famílias, cinco gêneros e 10 espécies (Tabela 1). A espécie dominante foi Hypsiboas albopunctatus, com 46,7\% dos indivíduos registrados. Sete espécies apresentaram abundância intermediária, entre 2,9 e 14,5\%, e duas espécies foram raras, Scinax fuscomarginatus e Leptodactylus cf. ocellatus, representando menos de $1 \%$ do total de indivíduos (Tabela 1, Figura 4a).

$\mathrm{Na}$ área preservada foram registrados 656 indivíduos pertencentes a quatro famílias, seis gêneros e 11 espécies. A espécie dominante foi Dendropsophus giesleri, com 36,0\% dos indivíduos registrados. 
Tabela 2. Diversidade de anfíbios anuros do ambiente de área alterada (açude, mata secundária) e área preservada (poça permanente, mata pouco perturbada) do Parque Estadual Carlos Botelho, SP, entre agosto de 1999 e julho de 2000: número de indivíduos, riqueza de espécies, riqueza estimada por Jacknife $1 \pm$ desvio-padrão, espécies dominantes e dominância observada nos dois ambientes; riqueza e dominância estimadas por rarefação na área preservada ( $\mathrm{N}=518$, intervalo de confiança de $95 \%$ ).

Table 2. Diversity of amphibian anurans from the undisturbed habitat (artificial pond, secondary forest) and preserved habitat (permanent pond, old-growth forest) of Parque Estadual Carlos Botelho, SP, between August 1999 and July 2000: number of individuals, species richness, richness estimated by Jacknife $1 \pm$ standard deviation, dominant species and dominance observed in the two habitats; richness and dominance estimated by rarefaction in preserved habitat $(\mathrm{N}=518,95 \%$ confidence interval).

\begin{tabular}{|c|c|c|}
\hline & \multicolumn{2}{|c|}{ Ambiente } \\
\hline & Área alterada & Área preservada \\
\hline Número de indivíduos & 518 & 656 \\
\hline Riqueza observada & 10 & 11 \\
\hline Riqueza estimada (rarefação; $\mathrm{n}=518$ ) & - & 10 a 11 \\
\hline Riqueza estimada (jacknife1) & $11,9 \pm 1,9$ & $12.9 \pm 1,4$ \\
\hline Espécie dominante & Hypsiboas albopunctatus & Dendropsophus giesleri \\
\hline Dominância observada & $46,7 \%$ & $36,0 \%$ \\
\hline Dominância estimada $(\mathrm{n}=518)$ & - & 34,0 a $37,8 \%$ \\
\hline
\end{tabular}
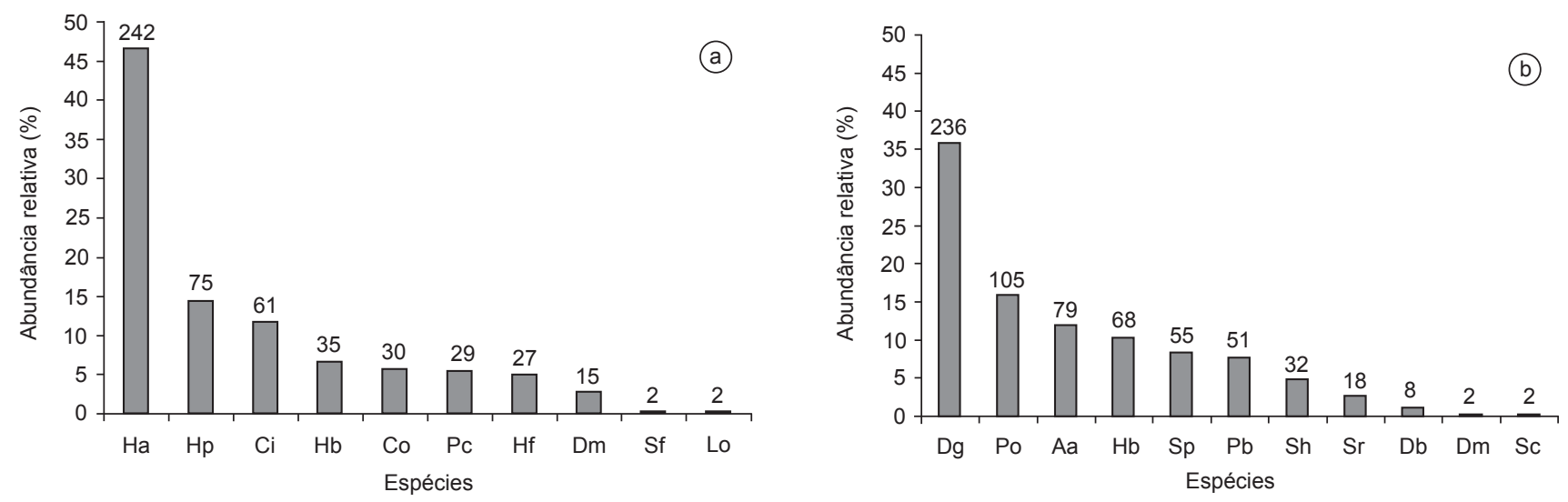

Figura 4. Abundância relativa das espécies de anfíbios anuros (porcentagem em relação ao número total de indivíduos) encontradas entre agosto de 1999 e julho de 2000 no Parque Estadual Carlos Botelho, SP, em dois ambientes: a) área alterada (açude em área de mata secundária) e; b) área preservada (poça permanente em mata pouco perturbada). Acima de cada coluna é indicado o número total de indivíduos registrados por espécie. Legenda: Aa = Aplastodiscus albosignatus; $\mathrm{Ci}=$ Chaunus ictericus $; \mathrm{Co}=$ C. ornatus $; \mathrm{Db}=$ Dendrophryniscus brevipollicatus $; \mathrm{Dg}=$ Dendropsophus giesleri $;$ Dm $=$ Dendropsophus minutus $; \mathrm{Ha}=$ Hypsiboas albopunctatus $; \mathrm{Hb}=H$. bischoff $; \mathrm{Hf}=H$. faber $; \mathrm{Hp}=H$. prasinus $;$ Lo $=$ Leptodactylus cf. ocellatus $; \mathrm{Pb}=$ Proceratophrys boiei $; \mathrm{Pc}=$ Physalaemus cuvieri; $\mathrm{Po}=$ Physalaemus olfersii $; \mathrm{Sc}=$ Scinax cf. catharinae $; \mathrm{Sf}=$ Scinax fuscomarginatus $; \mathrm{Sh}=$ S. hayii; $\mathrm{Sp}=$ Scinax perereca $; \mathrm{Sr}=$ Scinax $\mathrm{cf}$. ruber .

Figure 4. Relative abundance of amphibian anuran species (percentage from the total number of individuals) found between August 1999 and July 2000 in Parque Estadual Carlos Botelho, SP, in two habitats: A - disturbed habitat (artificial pond in secondary forest) and; B - preserved habitat (permanent pond in old-growth forest). The total number of individuals for each species is indicated above columns. Legend: Aa = Aplastodiscus albosignatus; Ci $=$ Chaunus ictericus; Co = C. ornatus; $\mathrm{Db}=$ Dendrophryniscus brevipollicatus $; \mathrm{Dg}=$ Dendropsophus giesleri $; \mathrm{Dm}=$ Dendropsophus minutus $; \mathrm{Ha}=$ Hypsiboas albopunctatus $; \mathrm{Hb}=$ H. bischoff $; \mathrm{Hf}=$ H. faber $; \mathrm{Hp}=$ H. prasinus $;$ Lo = Leptodactylus cf. ocellatus $; \mathrm{Pb}=$ Proceratophrys boiei $; \mathrm{Pc}=$ Physalaemus cuvieri $; \mathrm{Po}=$ Physalaemus olfersii $;$ $\mathrm{Sc}=$ Scinax cf. catharinae $; \mathrm{Sf}=$ Scinax fuscomarginatus $; \mathrm{Sh}=$ S. hayii $; \mathrm{Sp}=$ Scinax perereca $; \mathrm{Sr}=$ Scinax $\mathrm{cf}$. ruber .

Oito espécies apresentaram abundância intermediária, entre 1,2 e $16,0 \%$, e duas espécies foram raras, Dendropsophus minutus e Scinax cf. catharinae representando menos de $1 \%$ do total de indivíduos (Tabela 1, Figura 4b).

A área preservada apresentou riqueza observada de 11 espécies, valor superior ao registrado na área alterada (10 spp.), mas também maior número de indivíduos (Tabela 2). Entretanto, considerando o mesmo número de indivíduos nas duas áreas $(\mathrm{N}=518)$, o método de rarefação estimou entre 10 e 12 espécies na área preservada, ou seja, não significativamente diferente em relação à riqueza observada na área alterada. Mas as curvas de rarefação (Figura 5) ilustram maior riqueza de espécies na área preservada, tanto para a curva de rarefação baseada em número de amostras (Figura 5a) quanto para aquela baseada em número de indivíduos (Figura 5b). Para os dois ambientes, as curvas de rarefação de espécies não estabilizaram, ou seja, não atingiram um platô, indicando que não foram encontradas todas as espécies que ocorrem nesses ambientes (Figura 5). O estimador Jacknife de primeira ordem (Jack 1) foi o que apresentou o melhor comportamento ao longo da acumulação das amostras considerando as curvas de rarefação de estimadores nos dois ambientes (Figura 6). Este estimador indica que devem ocorrer na área preservada entre 12 e 14 espécies e na área alterada entre 10 e 14 espécies (Tabela 2, Figura 6).

A dominância observada foi menor na área preservada (36\%) em relação à área alterada (46,7\%; Tabela 2). A dominância estimada por rarefação para a área preservada, entre 34,0 e 37,8\%, considerando o 

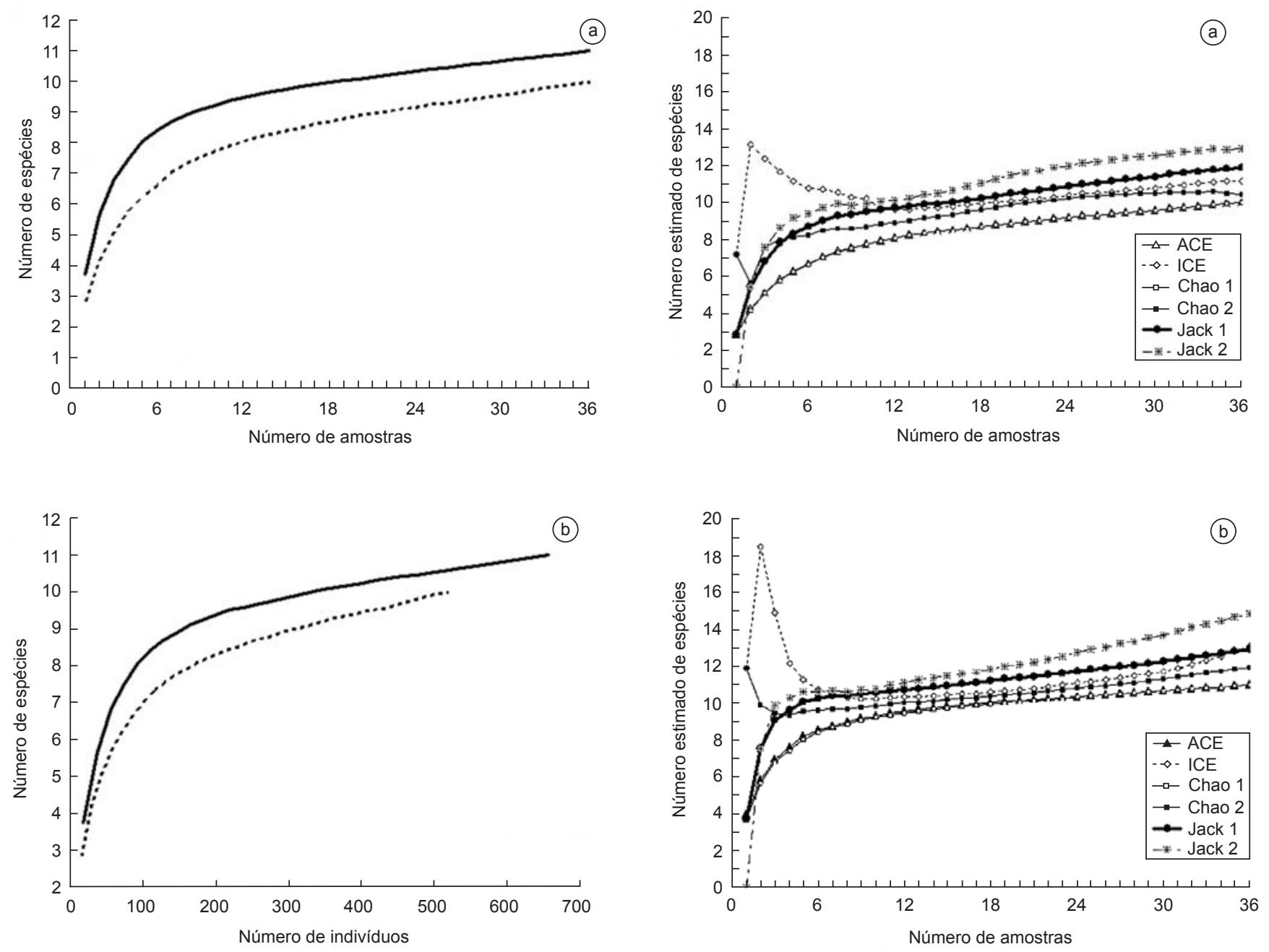

Figura 5. a) Curvas de rarefação de espécies da área alterada (linha pontilhada) e da área preservada (linha contínua), confeccionadas a partir de 1.000 aleatorizações na ordem das amostras (veja detalhes em Análise de Dados); e b) Curvas de rarefação de espécies da área alterada (linha pontilhada) e da área preservada (linha contínua) em relação ao número de indivíduos estimados a partir de 1.000 aleatorizações na ordem das amostras (veja detalhes em Análise de Dados).

Figure 5. a) Rarefaction curves of species from the disturbed habitat (dashed line) and from the preserved habitat (continuous line), constructed from 1000 randomizations on the order of samplings (see details in the text); and b) Rarefaction curves of species from the disturbed habitat (dashed line) and from the preserved habitat (continuous line) in relation to the number of individuals estimated from 1000 randomizations on the order of samplings (see details in the text).

mesmo número de indivíduos amostrados na área alterada ( $\mathrm{N}=518)$, foi significativamente menor (Tabela 2).

\section{Discussão}

Ao menos três fatores não mutuamente exclusivos e associados à alteração da cobertura vegetal original de Mata Atlântica podem ser responsáveis pelos diferentes padrões de composição e diversidade de espécies de anuros observados nos ambientes estudados: a) a capacidade das populações de algumas espécies em colonizar o ambiente de área alterada, originado por ação antrópica; b) tolerâncias fisiológicas distintas das espécies em relação às condições ambientais;

Figura 6. a) Curvas de rarefação de estimadores de riqueza de espécies da área alterada; e b) Curvas de rarefação de estimadores de riqueza de espécies da área preservada, confeccionadas a partir de 1.000 aleatorizações na ordem das amostras (veja detalhes em Análise de Dados).

Figure 6. a) Rarefaction curves of richness estimators form the disturbed habitat; and b) Rarefaction curves of richness estimators form the preserved habitat, constructed from 1000 randomizations on the order of samplings (see details in the text).

e c) dependência de microambientes específicos para a reprodução por algumas espécies das duas áreas.

Algumas espécies que ocorrem na área alterada (e.g., Hypsiboas albopunctatus, Leptodactylus cf. ocellatus e Physalaemus cuvieri) são ecologicamente generalistas e podem invadir ambientes alterados pelo homem (Heyer et al. 1990, Haddad \& Sazima 1992, Haddad 1998, Ramos \& Gasparini 2004, Haddad \& Prado 2005, Conte \& Rossa-Feres 2006). Essas espécies habitam formações vegetais originalmente abertas (cf. Brasileiro et al. 2005) e expandem as suas distribuições geográficas com os desmatamentos e conseqüente alteração da paisagem natural (Haddad 1998). Quando os ambientes alterados propiciam a estas espécies colonizadoras alimento abundante, abrigos e locais adequados para a reprodução, podem favorecer a permanência de suas populações (e.g., Meshaka 1996, Henderson \& Powell 2001). Em alguns casos, populações destas espécies podem atingir altos valores de dominância (e.g., Heinen 1992, Giaretta et al. 1999), como aquele observado para $H$. albopunctatus na área alterada. 
A estrutura da cobertura vegetal influencia a temperatura da água, a intensidade luminosa e a umidade próxima à superfície do solo (Bellis 1962, Crump 1971, Pough et al. 1977, Halverson et al. 2003, Felix et al. 2004). Ambientes que sofreram alterações antrópicas são caracterizados por vegetação mais esparsa e estão sujeitos a maior exposição solar, menor umidade e temperaturas mais elevadas quando comparados a áreas de mata preservada, com vegetação alta, densa e dossel fechado (Sexton et al. 1964, Crump 1971, Inger \& Colwell 1977, Pough et al. 1977). As características particulares de cada ambiente em relação a estes fatores, possivelmente determinam e limitam a distribuição das espécies presentes no PECB, uma vez que diferentes espécies de anuros respondem diferencialmente às condições ambientais, apresentando diferentes taxas de crescimento e desenvolvimento, além de exibirem graus distintos de resistência e tolerância a perda de água (e.g., Thorson \& Svihla 1943, Thorson 1955, Bellis 1962, Heatwole et al. 1968, Crump 1971, Snyder \& Weathers 1975, Pough et al. 1977, Christian et al. 1988, Giaretta et al. 1997, Werner \& Glennemeier 1999, Halverson et al. 2003, Felix et al. 2004). Espécies do gênero Chaunus, por exemplo, toleram maiores níveis de perda d'água em relação a espécies de outros grupos de anuros (cf. Thorson \& Svihla 1943, Thorson 1955). Isto pode explicar em parte a presença e a abundância das duas espécies deste gênero na área alterada, que não foram registradas na área preservada. Inger \& Colwell (1977) sugerem que a baixa previsibilidade da temperatura e da evaporação em áreas perturbadas, em relação às áreas pouco perturbadas na Thailândia, impedem a formação de diversas guildas da herpetofauna, o que resulta em baixa diversidade de espécies.

Em áreas florestais maduras e pouco perturbadas, considera-se que a fauna atinja um equilíbrio no número de espécies e nas relações entre riqueza e abundância (Scott-Jr 1976), sendo o oposto verdadeiro para ambientes em sucessão secundária (Heinen 1992). Portanto, a menor diversidade de espécies no ambiente alterado deve estar relacionada à lenta restauração da herpetofauna desde o período do desmatamento para a construção do açude (ca. 25 anos) até os dias de hoje (cf. Heinen 1992), além da presença de espécies invasoras típicas de área aberta.

A seleção de hábitats reprodutivos associada às limitações impostas por modos reprodutivos específicos pode restringir a utilização de um determinado hábitat (Collins \& Wilbur 1979, Bertoluci \& Rodrigues 2002). Algumas espécies restritas a área preservada possuem modos reprodutivos mais especializados. Por exemplo, Dendrophryniscus brevipollicatus desova dentro de bromélias (Carvalho 1949) e Aplastodiscus albosignatus possivelmente em tocas subterrâneas escavadas pelos machos (cf. Haddad \& Sawaya 2000, Pombal-Jr \& Gordo 2004). Diversas espécies de anuros com modos reprodutivos mais especializados e adaptadas a microambientes específicos para a reprodução, permanecem restritas a áreas de mata preservadas devido a ausência destes microambientes em áreas alteradas (Haddad 1998). Deste modo, a maior heterogeneidade ambiental dos ambientes florestais, em relação aos ambientes abertos, pode suportar uma maior riqueza de espécies por fornecer um maior número de microambientes disponíveis para a reprodução (e.g., Cardoso et al. 1989, Tocher 1998, Bernarde et al. 1999). Além disso, D. brevipollicatus, A. albosignatus e Scinax cf. catharinae são espécies intrinsecamente relacionadas ao Domínio Morfoclimático da Floresta Atlântica e, neste domínio, são exclusivas de hábitats florestados (Heyer et al. 1990, Guix et al. 1994). Portanto, a existência de áreas florestais pouco perturbadas, que incluam maior número de microambientes disponíveis para a reprodução são imprescindíveis para a sobrevivência destas espécies e para a manutenção da diversidade biológica (cf. Zimmermman \& Bierregaard 1986, Haddad 1998). Sugerimos que alterações adicionais da cobertura original de Mata Atlântica na reserva poderão resultar em um decréscimo na diversidade alfa (riqueza local de espécies), devido à eliminação de espécies ecologicamente dependentes de ambientes florestais, com conseqüente aumento na diversidade beta (substituição de espécies), resultante da colonização de espécies típicas de área aberta e ecologicamente mais generalistas. Provavelmente, também ocorreria um aumento na dominância, resultante da invasão destas espécies generalistas, como observado na área alterada.

$\mathrm{O}$ fato de nem todas as espécies que ocorrem nos dois ambientes terem sido capturadas (R.A. Moraes \& L.M. Castanho, dados não publicados), como também indicado pelas curvas de rarefação, mostra a importância da realização de estudos de longo prazo e da associação de diferentes métodos de amostragem para um inventário mais completo da anurofauna, sobretudo em áreas com elevada riqueza como a Floresta Atlântica (cf. Heyer et al. 1990, Conte \& Rossa-Feres 2006). Apesar de não terem sido capturadas todas as espécies que devem ocorrer nos dois ambientes, a riqueza foi ligeiramente maior na área preservada, embora a diferença não seja significativa. Possivelmente, a riqueza de espécies nos dois ambientes não foi significativamente diferente devido ao número relativamente baixo de espécies encontradas. Essa baixa riqueza faz com que o intervalo de confiança da estimativa seja grande em relação ao número total de espécies. Além disso, o açude da área alterada apresenta área muito maior (cerca de 40 vezes) em relação à poça permanente da área preservada. Áreas maiores suportam um maior número de espécies (MacArthur \& Wilson 1967). Assim, é provável que a diferença na riqueza de espécies entre os dois ambientes seja muito maior, quando considerados sítios reprodutivos de tamanho semelhante.

A riqueza ligeiramente maior e principalmente a menor dominância observada na área preservada, indicam ser esta uma área que apresenta maior diversidade de espécies em relação à área alterada. Assim, a presença de florestas pouco perturbadas na reserva é fundamental para a manutenção da diversidade da anurofauna local.

\section{Agradecimentos}

Somos gratos ao diretor José Luiz Camargo Maia e aos funcionários do Parque Estadual Carlos Botelho pelo apoio logístico durante as coletas de dados. A dois assessores anônimos e Dra. Denise C. Rossa-Feres pelas ótimas sugestões no manuscrito. Ao Prof. Dr. Célio F. B. Haddad pelo auxílio na identificação das espécies. Ao Instituto Florestal de São Paulo (IF) pela permissão da pesquisa em área sob sua gerência. RAM agradece ao Conselho Nacional de Desenvolvimento Científico e Tecnológico (PIBIC-CNPq) pela bolsa concedida e ao IBAMA pela concessão da licença de coleta ( ${ }^{\circ}$ 612/99).

\section{Referências Bibliográficas}

AZEVEDO-RAMOS, C. \& GALATTI, U. 2002. Patterns of amphibian diversity in Brazilian Amazonia: conservation implications. Biol. Conserv. 103(1):103-111.

BEGON, M., TOWNSEND, C.R. \& HARPER, J.L. 2006. Ecology: From individuals to ecosystems. 4 ed. Blackwell Scientific Publications, Oxford.

BELLIS, E.D. 1962. The influence of humidity on wood frog activity. Am. Midl. Nat. 68(1):139-148.

BERNARDE, P.S., KOKUBUM, M.N.C., MACHADO, R.A. \& ANJOS, L. 1999. Uso de hábitats naturais e antrópicos pelos anuros em uma localidade no Estado de Rondônia, Brasil (Amphibia: Anura). Acta. Amazon. 29(4):555-562.

BERTOLUCI, J. \& RODRIGUES, M.T. 2002. Utilização de habitats reprodutivos e micro-habitats de vocalização em uma taxocenose de anuros (Amphibia) da Mata Atlântica do sudeste do Brasil. Pap. Avulsos Zool. 42(11):287-297.

BLAUSTEIN, A.R. \& WAKE, D.B. 1995. The puzzle of declining amphibian populations. Sci. Am. 272(4):52-57. 
BRASILEIRO, C.A., SAWAYA, R.J., KIEFER, M.C. \& MARTINS, M. 2005. Amphibians of an open Cerrado fragment in southeastern Brazil. Biota Neotrop. 5(2): http://www.biotaneotropica.org.br/v5n2/pt/abstract?artic le+BN00405022005 (último acesso em 07/11/2006).

BURNHAM, K.P. \& OVERTON, W.S. 1979. Robust estimation of population size when capture probabilities vary among animals. Ecology 60(5): 927-936.

CALDWELL, J.P. 1996. Diversity of Amazonian anurans: the role of systematics and phylogeny in identifying macroecological and evolutionary patterns. In Neotropical biodiversity and conservation (A.C. Gibson, ed.). Occas. Publ. Mildred E. Mathias Bot. Gdn, Los Angeles, v.1, p.73-88.

CAMARGO, J.C.G., PINTO, S.A.F. \& TROPPMAIR, H. 1972. Estudo fitogeográfico e ecológico da Bacia Hidrográfica Paulista do Rio da Ribeira. Biogeografia 5:1-32

CARDOSO, A.J., ANDRADE, G.V. \& HADDAD, C.F.B. 1989. Distribuição espacial em comunidades de anfíbios (Anura) no sudeste do Brasil. Rev. Bras. Biol. 49(1):241-249.

CARVALHO, A.L. 1949. Notas sôbre os hábitos de Dendrophryniscus brevipollicatus Espada (Amphibia, Anura). Rev. Bras. Biol. 9(2):223-227.

CHRISTIAN, K.A., NUNEZ, F., CIOS, L. \& DIAZ, L. 1988. Thermal relations of some tropical frogs along an altitudinal gradient. Biotropica 20(3):236-239.

COLLINS, J.P. \& WILBUR, H.M. 1979. Breeding habits and habitats of the amphibians of the Edwin S. George Reserve, Michigan, with notes on the local distribution of fishes. Occas. Pap. Mus. Zool. Univ. Mich. 686:1-34.

COLLINS, J.P. \& STORFER, A. 2003. Global amphibian declines: sorting the hypotheses. Divers. Distrib. 9:89-98.

COLWELL, R.K. 2005. EstimateS: Statistical estimation of species richness and shared species from samples. Version 7.5. Persistent URL <purl. oclc.org/estimates $>$.

CONTE, C.E. \& ROSSA-FERES, D.C. 2006. Diversidade e ocorrência temporal da anurofauna (Amphibia, Anura) em São José dos Pinhais, Paraná, Brasil. Rev. Bras. Zool. 23(1):162-175.

CRUMP, M.L. 1971. Quantitative analysis of the ecological distribution of a tropical herpetofauna. Occas. Pap. Univ. Kansas Mus. Nat. Hist. 3:1-62.

DUELLMAN, W.E. 1988. Patterns of species diversity in anuran amphibians in the American tropics. Ann. Missouri. Bot. Gard. 75(1):79-104

DUELLMAN, W.E. 1999. Global distribution of amphibians: patterns, conservation and future challenges. In Patterns of distribution of amphibians: A global perspective (W.E. Duellman, ed.). The John Hopkins University Press, Baltimore \& London, p.1-30.

EHRLICH, P.R. \& WILSON, E.O. 1991. Biodiversity studies: science and policy. Science 253(5021):758-762.

ETEROVICK, P. C., CARNAVAL, A. C. O. Q., BORGES-NOJOSA, D. M., SILVANO, D. L., SEGALLA, M. V. \& SAZIMA I. 2005. Amphibian Declines in Brazil: An Overview. Biotropica 37(2):166-179.

FELIX, Z.I., WANG, Y. \& SCHWEITZER, C.J. 2004. Relationships between herpetofaunal community structure and varying levels of overstory tree retention in northern Alabama: first-year results. In Proceedings of the $12^{\text {th }}$ biennial southern silvicultural research conference (K.F. Connor, ed.). Gen. Tech. Rep., Asheville, p.7-10.

FERRAZ, L.P.M. \& VARJABEDIAN, R. 1999. Evolução histórica da implantação e consolidação das informações disponíveis sobre o Parque Estadual Carlos Botelho. Secretaria do Meio Ambiente, São Paulo.

GASCON, C., WILLIAMSON, G.B. \& FONSECA, G.A.B. 2000. Receding forest edges and vanishing reserves. Science 288(5470):1356-1358.

GIARETTA, A.A., SAWAYA, R.J., MACHADO, G., ARAÚJO, M.S., FACURE, K.G., MEDEIROS, H.F. \& NUNES, R. 1997. Diversity and abundance of litter frogs at altitudinal sites at Serra do Japi, southeastern Brazil. Rev. Bras. Zool. 14(2):341-346.

GIARETTA, A.A., FACURE, K.G., SAWAYA, R.J., MEYER, J.H.M. \& CHEMÍN, N. 1999. Diversity and abundance of litter frogs in a montane forest of southeastern Brazil: seasonal and altitudinal changes. Biotropica 31(4):669-674.
GOTELLI, N.J. \& COLWELL, R.K. 2001. Quantifying biodiversity: procedures and pitfalls in the measurement and comparison of species richness. Ecol. Lett. 4:379-391.

GOTELLI, N.J. \& ENTSMINGER, G.L. 2001. EcoSim: Null models software for ecology. Version 7.0. Acquired Intelligence Inc. \& Kesey-Bear. http://homepages.together.net/ gentsmin/ecosim.htm

GUIX, J.C., NUNES, V.S. \& MIRANDA, J.R. 1994. Autochthonous and colonizing species of frogs in "Carlos Botelho" State Reserve, Southeastern Brazil. Bol. Asoc. Herpetol. Esp. 5:8-13.

GUIX, J.C., MONTORI, A., LlORENTE, G.A., CARRETERO, M.A. \& SANTOS, X. 1998. Natural history and conservation of bufonids in four Atlantic Rainforest areas of southeastern Brazil. Herpetol. Nat. Hist. 6(1):1-12.

HADDAD, C.F.B. 1998. Biodiversidade dos anfíbios no Estado de São Paulo. In Biodiversidade do Estado de São Paulo, Brasil: síntese do conhecimento ao final do século XX (R.M.C. Castro, ed.). Editora Fapesp, São Paulo, v.6, p.17-26.

HADDAD, C.F.B. \& PRADO, C.P.A. 2005. Reproductive modes in frogs and their unexpected diversity in the Atlantic Forest of Brazil. BioScience 55(3):207-217.

HADDAD, C.F.B. \& SAZIMA, I. 1992. Anfíbios anuros da Serra do Japi. In História natural da Serra do Japi: Ecologia e preservação de uma área florestal no sudeste do Brasil (L.P.C. Morellato, ed.). Editora da Unicamp/FAPESP, Campinas, p.188-211.

HADDAD, C.F.B. \& SAWAYA, R.J. 2000. Reproductive modes of Atlantic Forest hylid frogs: a general overview and the description of a new mode. Biotropica 32(4b):862-871.

HALVERSON, M.A., SKELLY, D.K., KIESECKER, J.M. \& FREIDENBURG, L.K. 2003. Forest mediated light regime linked to amphibian distribution and performance. Oecologia 134(3):360-364.

HEATWOLE, H., AUSTIN, S.B. \& HERRERO, R. 1968. Heat tolerances of tadpoles of two species of tropical anurans. Comp. Biochem. Physiol. 27:807-815.

HEINEN, J.T. 1992. Comparisons of the leaf litter herpetofauna in abandoned cacao plantations and primary rain forest in Costa Rica: some implications for faunal restoration. Biotropica 24(3):431-439.

HENDERSON, R.W. \& POWELL, R. 2001. Responses by the West Indian Herpetofauna to human-influenced resources. Caribb. J. Sci. 37(1-2):41-54.

HEYER, W.R., RAND, A.S., CRUZ, C.A.G. \& PEIXOTO, O.L. 1988. Decimations, extinctions, and colonizations of frog populations in southeast Brazil and their evolutionary implications. Biotropica 20(3):230-235.

HEYER, W.R., RAND, A.S., CRUZ, C.A.G., PEIXOTO, O.L. \& NELSON, C.E. 1990. Frogs of Boracéia. Arq. Zool. 31:231-410.

HEYER, W.R., DONNELLY, M.A., MCDIARMID, R.W., HAYEK, L.C. \& FOSTER, M.S. 1994. Measuring and monitoring biological diversity: standard methods for amphibians. Smithsonian Institution Press, Washington and London.

HIROTA, M.M. 2005. Monitoramento da cobertura da Mata Atlântica brasileira. In Mata Atlântica: Biodiversidade, ameaças e perspectivas (C. Galindo-Leal \& I.G. Câmara, eds.). Fundação SOS Mata Atlântica, São Paulo, p.60-65.

HOULAHAN, J.E., FINDLAY, C.S., SCHMIDT, B.R., MEYER, A.H. \& KUZMIN, S.L. 2000. Quantitative evidence for global amphibian population declines. Nature 404(6779):752-755.

INGER, R. F. \& COLWELL, R. K. 1977. Organization of contiguous communities of amphibians and reptiles in Thailand. Ecol. Monogr. 47:229-253

KÖEPPEN, W. 1948. Climatología. Fundo de Cultura Economica, México.

KREBS, C.J. 2000. Ecological Methodology. 2 ed. Harper and Row Publishers, New York.

LIEBERMAN, S.S. 1986. Ecology of the leaf litter herpetofauna of a Neotropical Rain Forest: La Selva, Costa Rica. Acta. Zool. Mex. 15:1-71.

MACARTHUR, R.H. \& WILSON, E.O. 1967. The theory of island biogeography. Princeton University Press, Princeton. 
MACHADO, R.A., BERNARDE, P.S., MORATO, S.A.A. \& ANJOS, L. 1999. Análise comparada da riqueza de anuros entre duas áreas com diferentes estados de conservação no município de Londrina, Paraná, Brasil (Amphibia, Anura). Rev. Bras. Zool. 16(4):997-1004.

MAGURRAN, A.E. 1988. Ecological diversity and its measurement. Princeton University Press, Princeton.

MELO, A.S., PEREIRA, R.A.S., SANTOS, A.J., SHEPHERD, G.J., MACHADO, G., MEDEIROS, H.F. \& SAWAYA, R.J. 2003. Comparing species richness among assemblages using sample units: Why not use extrapolation methods to standardize different sample sizes? Oikos 101:398-410.

MESHAKA Jr, W.E. 1996. Diet and the colonization of buildings by the Cuban treefrog, Osteopilus septentrionalis (Anura: Hylidae). Caribb. J. Sci. 32(1):59-63

MORELLATO, L.P.C. \& HADDAD, C.F.B. 2000. Introduction: The Brazilian Atlantic Forest. Biotropica 32(4):786-792.

MYERS, N. \& KNOLL, A.H. 2001. The biotic crises and the future of evolution. PNAS. 98(10):5389-5392.

MYERS, N., MITTERMEIER, R.A., MITTERMEIER, C.G., FONSECA, G.A.B. \& KENT, J. 2000. Biodiversity hotspots for conservation priorities. Nature 403:853-858.

NECKEL-OLIVEIRA, S., MAGNUSSON, W.E. \& LIMA, A.P. 2000. Diversity and distribution of frogs in an Amazonian savanna in Brazil. Amphibia-Reptilia 21(3):317-326.

OLIVEIRA-FILHO, A.T. \& FONTES, M.A.L. 2000. Patterns of floristic differentiation among Atlantic Forests in southeastern Brazil and the influence of climate. Biotropica 32(4b):793-810.

OLMOS, F. \& GALETTI, M. 2004. A conservação e o futuro da Juréia: isolamento ecológico e impacto humano. In Estação Ecológica JuréiaItatins: Ambiente físico, flora e fauna (O.A.V. Marques \& W. Duleba, eds.). Editora Holos, Ribeirão Preto, p.360-377.

PEARMAN, P.B. 1997. Correlates of amphibian diversity in an altered landscape of Amazonian Ecuador. Conserv. Biol. 11(5):1211-1225.

PECHMANN, J.H.K. \& WILBUR, H.M. 1994. Putting declining amphibian populations in perspective: natural fluctuations and human impacts. Herpetologica 50(1):65-84.

POMBAL Jr, J.P. \& GORDO, M. 2004. Anfíbios anuros da Juréia. In Estação Ecológica Juréia-Itatins: Ambiente físico, flora e fauna (O.A.V. Marques \& W. Duleba, eds.). Editora Holos, Ribeirão Preto, p.243-256.

POMBAL Jr, J.P. \& HADDAD, C.F.B. 1999. Frogs of the genus Paratelmatobius (Anura: Leptodactylidae) with descriptions of two new species. Copeia 1999(4):1014-1026.
POUGH, F.H., STEWART, M.M. \& THOMAS, R.G. 1977. Physiological basis of habitat partitioning in Jamaican Eleutherodactylus. Oecologia 27:285-293.

RAMOS, D.A. \& GASPARINI, J.L. 2004. Anfíbios do Goiapaba-Açu, Fundão, Estado do Espírito Santo. Gráfica Santo Antônio, Espírito Santo.

SANDERS, H. 1968. Marine benthic diversity: a comparative study. Am. Nat. 102:243-282.

SCOTT-JR, N.J. 1976. The abundance and diversity of the herpetofaunas of tropical forest litter. Biotropica 8(1):41-58.

SEXTON, O.J., HEATWOLE, H. \& KNIGHT, D. 1964. Correlation of microdistribution of some Panamanian reptiles and amphibians with structural organization of the habitat. Caribb. J. Sci. 4(1):261-295.

SILVA, M.C. \& CASTELETI, C.H.M. 2005. Estado da biodiversidade da Mata Atlântica brasileira. In Mata Atlântica: Biodiversidade, ameaças e perspectivas (C. Galindo-Leal \& I.G. Câmara, eds.). Fundação SOS Mata Atlântica, São Paulo, p.43-59.

SNYDER, G.K. \& WEATHERS, W.W. 1975. Temperature adaptations in amphibians. Am. Nat. 109:93-101.

THORSON, T.B. 1955. The relationship of water economy to terrestrialism in amphibians. Ecology 36(1):100-115.

THORSON, T. \& SVIHLA, A. 1943. Correlation of the habitats of amphibians with their ability to survive the loss of body water. Ecology 24(3): 374-381.

TOCHER, M. 1998. Diferenças na composição de espécies de sapos entre três tipos de floresta e campo de pastagem na Amazônia Central. In Floresta Amazônica: Dinâmica, regeneração e manejo (C. Gascon \& P. Moutinho, eds.). Ministério da Tecnologia e Ciência, Manaus, p.219-232.

WERNER, E.E. \& GLENNEMEIER, K.S. 1999. Influence of forest canopy cover on the breeding pond distributions of several amphibian species. Copeia 1999(1):1-12.

WEYGOLDT, P. 1989. Changes in the composition of mountain stream frog communities in the Atlantic mountains of Brazil: frogs as indicators of environmental deteriorations? Stud. Neotrop. Fauna Env. 24(4):249-255.

ZAHER, H., AGUIAR, E. \& POMBAL-JR., J.P. 2005. Paratelmatobius gaigeae (Cochran, 1938) re-discovered (Amphibia, Anura, Leptodactylidae). Arq. Mus. Nac. 63(2):321-328.

ZIMMERMAN, B.L. \& BIERREGAARD, R.O. 1986. Relevance of the equilibrium theory of island biogeography and species-area relations to conservation with a case from Amazonia. J. Biogeogr. 13:133-143. 